\title{
THE EFFECT OF DIFFRACTION ON THE REDISTRIBUTION OF WAVE ENERGY IN THE LEE OF AN OVERTOPPING TYPE WAVE ENEGRY CONVERTER ARRAY
}

\author{
Kieran Monk ${ }^{1}$, Qingping Zou ${ }^{2}$ and Daniel Conley ${ }^{3}$
}

\begin{abstract}
A computationally efficient approximate analytical solution based on the classic solution for diffraction about a semi-infinite breakwater, is developed to assess the wave energy shadow down-wave of a single row array of overtopping type wave energy converters approximated as partially transmitting breakwater segments. The approximations associated with the solution are mitigated by a comparison with the mathematically exact but computationally expensive boundary element method for the same problem. The approximate analytical solution is compared with a hypothetical solution where diffraction is not accounted for to quantify the net re-distribution of wave energy by the diffraction mechanism with increasing down-wave distance from the array.
\end{abstract}

Keywords: wave energy converter; wave energy shadow; scattering; diffraction; breakwater, wave dragon;

\section{Introduction}

The Wave Dragon is a floating overtopping type wave energy converter (WEC). The basic composition of the device is a floating reservoir with wings that focus energy towards a ramp on the leading edge of the reservoir that forces the focused incident waves to run-up and overtop. The water that overtops into the elevated reservoir has a positive potential energy and returns to the sea through low head turbines to generate electricity. The remaining incident wave energy not captured by the device will be transmitted below the device, reflected from the face of the device or radiated from the motion of the floating device in heave. These ratios will be dependent on the incident wave height, frequency and direction as well as the specific geometry of the device and mooring system. If we ignore the motion associated with a floating device then a dissipative porous breakwater segment can be used to approximate the overtopping type WEC device because it can be configured to reflect, dissipate and transmit the same ratios of incident wave energy. However, the geometrical intricacies of the device, namely the focusing wings, are not considered when the device is approximated as a breakwater segment. Nøgaard and Anderson (2012) compare the disturbed wave field in the lee of a Wave dragon array using the Boussinesq wave propagation model Mike21. The energy extraction and wave structure interactions of the WEC devices were approximated in the model using dissipative sponge layers. The geometry of the sponge layers was configured for both a realistic geometrical representation of the Wave Dragon device with spatially variable dissipation (for wings and main body), and for a simplified device that is essentially just a plain uniformly dissipating breakwater segment or homogenous sponge layer block. They show that at a distance from the array greater than $2 \mathrm{~km}$ (and probably much closer) accounting for the geometrical intricacies of the device has little impact on the resultant disturbed wave field.

The extraction of wave energy from the system and reflection from the device will result in a wave energy deficit down wave in the immediate lee of the device. With increasing distance from the device wave directional spreading will re-distribute the remaining forward propagating wave energy so that the maximum reduction in wave energy at any one point, compared to the undisturbed wave field, will reduce with increasing distance from the device. In addition the energy extraction from the device will result in a sharp gradient in the surface elevation along the geometrical shadow line of the device. This will induce the diffraction effect. Diffraction will also act to re-distribute wave energy but the net effect is less obvious than the influence of directional spreading and although diffraction is accounted for (Venugopal and Smith 2007, Beels et al. 2010 and Nøgaard and Anderson 2012), the specific net influence of diffraction on the wave energy shadow has received less attention.

In this study we investigate the effect of diffraction on the re-distribution of wave energy from a single row array of overtopping type wave energy converters approximated as dissipative breakwater segments using an approximate analytical solution. The approximate analytical solution used in this study is highly computationally efficient, even using serial programming on a basic PC, making it useful for investigating random wave sea states for large domains. This makes it suitable for studying a full wave climate for arrays placed far offshore. The analytical solution for diffraction about a reflecting or absorbing semi-infinite breakwater was superposed to give the approximate solution of the diffracted wave field about a reflecting or absorbing segmented breakwater series. The adapted analytical solution for a fully absorbing breakwater predicts the same locations of the interference maximas and minimas as REF/DIF, while thatfor a fully reflecting breakwater predicted the same locations of the interference maxima and minimas as FUNWAVE (Monk et al 2012b).

\footnotetext{
${ }^{1}$ School of Marine Science and Engineering, University of Plymouth, Plymouth, PL48AA, UK

2 Department of Civil and Environmental Engineering, University of Maine, Orono, ME 04469, USA

${ }^{3}$ School of Marine Science and Engineering, University of Plymouth, Plymouth, PL48AA, UK
} 
Using the boundary element method (BEM) McIver (2005) provides a mathematically exact analytical solution for the transmitted and scattered wave energy field about a single row of permeable breakwater segments. This however is comparatively computationally expensive making it less suitable for assessing large domains for a full range of random sea states. It can however be used to mitigate, in part, the approximations associated with the approximate analytical solution. The re-distribution of wave energy when diffraction and directional spreading are considered is compared to the un-realistic case where only directional spreading is considered (no-diffraction) to assess the contribution of the diffraction effect on the re-distribution of wave energy in the lee of the array up to the very far field.

The ability to predict the re-distribution of wave energy in the lee of a WEC array and hence the progressive evolution of the wave energy shadow with increasing distance from the array towards the coast is of topical interest due to the significant stake-holder concerns about the potential impacts of offshore WEC installations. Waves play an important role in mass transport, assist in mixing and force sediment transport as well as providing a recreational medium for water users and in particular surfers, who can be very vocal in their concerns about potential wave height reductions.

\section{Method}

A number of options are available for assessing the transmitted and scattered wave field about an array of overtopping type wave energy converters approximated as dissipative segments. Miller et al. (2007) use dissipative regions in the phase averaged spectral wave models SWAN to simulate wave energy conversion. The model is computationally efficient and is useful for quickly assessing large domains for a wide range of sea states. However the diffraction effect is only weakly approximated and because the model does not resolve phase, interference of scattered waves is not accounted for. Time-dependent wave propagation models based on the mild slope equations can resolve phase and hence account for the diffraction and interference as demonstrated in Beels et al. (2010) using MILDwave. However time-dependent mild slope models are computationally expensive making the assessment of large domains for a wide number of high resolution random sea states more difficult. They also can incur stability issues and reflections at the domain boundary must be handled. The same applies to models based on the Boussinesq equations such as Mike21 which have the added benefit of accounting for non-linear effects. Mike21 is used in the wave shadow studies of Venugopal and Smith (2007) and Nøgaard and Anderson (2012).

In this study we propose an alternative method for scaling the wave energy shadow in the lee of a single row array of overtopping type wave energy converters, and use this method, specifically, to investigate the role of diffraction on energy re-distribution. This is achieved using an existing approximate analytical solution for the reflected, transmitted, dissipated and scattered wave field about a porous breakwater segment which we extend to consider a single row series of permeable breakwater segments that we will use to approximate an array of overtopping type WECs. The approximations associated with this solution are investigated in part with a comparison with the more computationally expensive but mathematically exact analytical solution for the wave field about a segmented permeable breakwater series using the BEM, as presented in McIver (2005).

Penney and Price (1952) provide an analytical solution for the diffracted wave field about a solid semiinfinite breakwater based on the Sommerfield (1886), solution using Fresnel integrals for obliquely incident monochromatic waves. They also provide approximate solutions for the diffracted wave field about a gap in an infinite breakwater and a detached breakwater segment by superimposing two semi-infinite breakwaters, for waves arriving with normal incidents. Silverster and Lim (1968) suggest that a partially reflecting semi-infinite breakwater can be approximated by applying coefficients to the components that describe the reflected wave and the diffracted wave of the reflected wave. Hotta (1978) adopt a similar method and apply transmission coefficient to provide an approximate solution for a partially reflecting and transmitting semi-infinite breakwater. They also extended the Penney and Price (1952) approximate solution for the detached breakwater segment to account for partial transmission and reflection by superimposing the solution for a breakwater gap with a smaller incident wave relative to the transmission through the breakwater segment. Ou et al. (1988) extend the solution further to account for refraction and show that the solution compares reasonably well in the near field with experimental data for a single detached permeable breakwater on a sloping bathymetry. Kim and Lee (2010) extend the approximate detached breakwater solution to account for obliquely incident waves by applying a phase shift term to the diffracted wave components to account for the difference in phase of the incident plane wave that drives the system, as it arrives at the opposing tips of the breakwater segment. We 
suggest that an approximate solution for the reflected, transmitted and diffracted wave field about a series of breakwater segments can be made with multiple superpositions of the detached breakwater solution. Oblique incident waves are permitted if the resultant solution is extended further to account for the phase shift of incident waves arriving at the tips of the other breakwater segments of the series (relative to the co-ordinate centre) in the same manner as Kim and Lee (2010).

This method has a number of approximations associated with it, the most apparent result of which is a discontinuity along the plane of the array. Specifically the approximations are that waves diffracting from one breakwater tip do not register the re-opening of the breakwater past the opposing tip, nor do they register the presence of the other breakwater segments in the series and as such secondary diffraction is not considered. As the diffracted waves are essentially radiation waves (weighted in the direction of propagation) the influence of secondary diffraction waves in the moderate to far-field $(y>50 \lambda)$ is expected to be minimal, which is the primary focus region of this study. We investigate this approximation further in the validation section with a comparison to the mathematically exact BEM of McIver (2005).

In addition the method of applying transmission and reflection coefficients to the terms of the full solution that describe the incident wave and diffraction of the incident wave, as well as the reflection and diffraction of the reflected wave, respectively, assumes that the incident and reflected wave components can be varied independently of each other. This is not mathematically accurate as Daemerich and Kohlhase (1978) show with a comparison of the approximate solution of a fully dissipating semi-infinite breakwater to experimental data and the mathematically exact solution of diffraction at the end of a guide wall wedge (Mitsui and Murakami 1967). They found that the approximate solution for a fully dissipative breakwater significantly under-predicts the wave height in the immediate lee of the breakwater but converges to the exact solution (guide wall wedge) with increasing distance from the breakwater. So this approximation seems reasonable in the far field which is the primary region of focus for this study. We investigate this approximation further in the validation section with a comparison to the mathematically exact boundary element method for partial transmission. However, breakwaters with high dissipation (our approximation of wave energy conversion) cannot be considered with the BEM in its published form in McIver (2005) and this approximation will remain un-validated.

\section{APPROXIMATE ANALYTICAL SOLUTION}

The derivation of the approximate analytical solution for a single detached permeable breakwater sections is lengthy and is given in full in Monk et al. (2002). The solution is the extension of the solution given in Hotta (1978) which it's self is ultimately an extension of the solution given in Penney and Price (1952) for a detached breakwater segment. The solution given in Monk et al. (2012) describes the disturbance coefficient, which is the ratio of the resultant wave height at a point in the domain $\mathrm{H}\left(r_{0}, \theta_{0}\right)$ and the incident wave height $H_{0}$, so that $K_{d}=\mathrm{H}\left(r_{0}, \theta_{0}\right) / H_{0}$, for a series of transmitting reflecting and dissipating breakwater segments for oblique incident waves. This is achieved by superposing the solution of the single segment to build up an array of multiple segments in a single row. The relative phase shift of the incident wave arriving at each segment tip in the series is accounted for by factoring in the difference in path length relative to one reference breakwater segment. We will not reiterate the solution here other than a few key points.

In the approximate analytical solution reflection and transmission coefficients are applied to the terms that describe the reflected and transmitted wave components, and this is used to define the dissipation in the structure. $c_{\rho}$ is the reflection coefficient ( $c_{\rho}=1$ for total reflection, and $c_{\rho}=0$ for zero reflection), and $c_{\iota}$ is the transmission coefficient $\left(c_{\iota}=1\right.$ for total transmission, and $c_{\iota}=0$ for zero transmission). For a partially transmitting breakwater (no dissipation) $c_{\rho}+c_{\iota}=1$, and for a dissipating breakwater $c_{\rho}+c_{\iota}<1$. This assumes no phase change of the wave passing through the breakwater (or below the WEC device as we intend to use the solution). For a permeable breakwater made from a porous medium we can adopt the complex reflection and transmission coefficients described in Yu and Togashi (1996) which is reiterated in Bowen and McIver (2000) (for consistency with the following section) as;

$$
\begin{aligned}
& c_{\rho}=\frac{-i k \sin \Theta_{0}}{2 \beta-i k \sin \Theta_{0}} \\
& c_{\iota}=\frac{2 \beta}{2 \beta-i k \sin \Theta_{0}}
\end{aligned}
$$


where $\Theta_{0}$ is the incident wave direction

$$
\beta=\frac{-i \epsilon}{b(f-i s)}
$$

where $\epsilon$ is the porosity, $f$ is a non-dimensional friction coefficient, $s$ is the non-dimensional inertia coefficient and $b$ is the physical thickness of the breakwater (although this is considered to be zero in the calculation domain)

$k$ is the wave number and is found from the dispersion relationship;

$$
\omega^{2}=g k \tanh (k h)
$$

where $\omega$ is the angular frequency given by $2 \pi / T$, where $T$ is the wave period, $g$ is the acceleration due to gravity and $h$ is the water depth.

The approximate analytical solution is solved using the Fresnel integrals $C\left(\sigma_{z}\right)$ and $S\left(\sigma_{z}\right)$. One might consider using the McCormick and Kraemer (2002) polynomial approximations for the Fresnel integrals, given in Eq. (5) and (6), which provide excellent accuracy and reduces the computational effort by approximately $90 \%$. This is very important when numerous calculations are required to statistically represent directional and spectral wave conditions which will be introduced later. All results presented in this article associated with the approximate analytical solution where calculated using these polynomial approximations.

$$
\begin{aligned}
& C\left(\sigma_{z}\right)=-C\left(-\sigma_{z}\right) \approx \frac{1}{2}+\frac{\left(1+0.926 \sigma_{z}\right) \sin \left(\frac{\pi \sigma_{z}{ }^{2}}{2}\right)}{2+1.792 \sigma_{z}+3.103 \sigma_{z}{ }^{2}}-\frac{\cos \left(\frac{\sigma_{z}{ }^{2}}{2}\right)}{2+4.142 \sigma_{z}+3.492 \sigma_{z}{ }^{2}+6.670 \sigma_{z}{ }^{3}}+\epsilon(\sigma) \\
& S\left(\sigma_{z}\right)=-S\left(-\sigma_{z}\right) \approx \frac{1}{2}-\frac{\left(1+0.926 \sigma_{z}\right) \cos \left(\frac{\sigma_{z}{ }^{2}}{2}\right)}{2+1.792 \sigma_{z}+3.103 \sigma_{z}{ }^{2}}-\frac{\sin \left(\frac{\sigma_{z}{ }^{2}}{2}\right)}{2+4.142 \sigma_{z}+3.492 \sigma_{z}{ }^{2}+6.670 \sigma_{z}{ }^{3}}+\epsilon(\sigma)
\end{aligned}
$$

where $\sigma_{z}$ and $\sigma_{z}{ }^{\prime}$ are the upper limits of the Fresnel integrals relative to breakwater tip number $z$ and the remainder for both equations $\epsilon(\sigma) \leq 0.002$

\section{BEM SOLUTION}

McIver (2005) provides a solution for the disturbed wave field about a series of thin permeable breakwater segments composed of discreet boundary elements based on linear wave theory. The boundary conditions of the permeable breakwater are described by the formulation of Sollitt and Cross (1972) for time-harmonic motion in a porous medium. The problem is solved with an integral equation and an application of the Green's theorem. The derivation of the solution is lengthy and challenging. Readers should refer to this paper for details of derivation. The BEM solution is only used in this study to mitigate some of the approximations associated with the approximate analytical solution and has not been modified.

Permeability of the breakwater is accounted for by a jump in potential across the breakwater segment which is described by the complex parameter $\beta$ given in Eq. (3) which incorporates the physical properties of the porous medium being modeled. The $\beta$ term is used to specify the reflection, transmission and dissipation characteristics of the permeable breakwater going from full reflection to near full transmission. Only partial dissipation can be achieved through the friction and inertia terms. As discussed in Yu and Togashi (1996) the reflected and transmitted wave incurs a phase change due to the representation of the inertia effects of the flow in a porous medium. The transmission of waves below an overtopping type WEC is not expected to incur the same phase change as it is not a porous medium.

The BEM method is mathematically exact and is based on the actual boundary conditions of the breakwater series and considers the influence the multi breakwater system as a whole. It is however comparatively computationally more expensive then the approximate analytical solution used here and is not practical for assessing large domains with high resolution random wave states. The computational effort for a full large 
domain at a given discreet wave height, period and direction combination is reasonable and also for domain transects for a random sea state. Also in its published form the McIver BEM method cannot consider high energy dissipation which we intend to use as an approximation of wave energy conversion. The present approximate analytical solution is computationally efficient making it suitable for assessing large domains for a large number of high resolution random sea states. As the focus of this investigation is to assess the influence of diffraction on the evolution of the wave energy shadow from a WEC array up to the far field, it is necessary to use the approximate analytical solution (to assess the whole domain), but it is important to validate the solution with a comparison to the mathematically exact BEM method. The approximations associated with the approximate analytical solution that can be validated with the BEM method are; the omission of secondary diffraction effects between opposing breakwater tips and other breakwater segments in the series. The BEM method can also be used to partially validate the approximations associated with the Hotta (1978) application of reflection and transmission coefficients to the Penney and Price (1952) solution that are not strictly based on the breakwater boundary conditions. However the dissipation condition when $c_{\rho}+c_{\iota} \neq 1$ cannot be validated by the BEM method in it's published form and we can only assume that the accuracy will be comparible to the validation of partial transmission when $c_{\rho}+c_{\iota}=1$. This is a point of caution and should receive further attention.

\section{NO-DIFFRACTION SOLUTION}

To isolate the effect of diffraction on the re-distribution of wave energy we must consider the hypothetical scenario where diffraction does not occur. This is very simple to achieve as the wave disturbance coefficient in the geometrical shadow of each device will be zero regardless of distance from the device and will be equal to unity at all other locations. This is similar to how a phase averaged model like SWAN would interpret the wave energy shadow provided that the diffraction approximation is off the domain has constant bathymetry and no friction is considered (Monk et al 2012 b). This is shown by Black (2007) with a comparison between what is essentially described above (no-diffraction solution) and the results of the Miller et al. (2007) using SWAN to assess the wave shadow for the wave hub project.

\section{RANDOM WAVES}

A real sea state is not monochromatic and the JONSWAP period spectrum $S(T)$ is one way to describe a spectral wave climate, statistically. The JONSWAP spectrum can be expressed as;

$$
S(T)=\frac{\alpha g^{2} T^{5}}{(2 \pi)^{4}} \exp \left[-\frac{5}{4}\left(\frac{T_{p}}{T}\right)^{-4}\right] \gamma^{q}
$$

where $T$ is the period, $T_{p}$ is the peak period of the spectrum, $\alpha$ is the Phillip's constant $(\alpha=0.0081), \gamma$ is the peak enhancement factor and $q=\exp \left[-\left((1 / T)-\left(1 / T_{p}\right)\right)^{2} T_{p}{ }^{2} / 2 \sigma^{2}\right]$, with $\sigma=0.07$ when $T \geq T_{p}$ and $\sigma=0.09$ when $T<T_{p}$.

The period spectrum function $S(T)$ can be scaled to a probability density function $P(T)$, by;

$$
P(T)=S(T) / \int_{0}^{\infty} S(T) d T
$$

where $\frac{1}{\int_{0}^{\infty} S(T) d T}$ is the normalising coefficient, and $\int_{0}^{\infty} P(T) d T=1$

The JONSWAP spectrum can be used to find the statistical mean diffracted wave field about the array under spectral wave conditions in the following manner. The wave energy field for each integer period component $T[s]$ of the period spectrum is multiplied by the respective $P(T)$ value for that period to give the scaled diffracted wave energy field for that particular value of $T[s]$. As $\int_{0}^{\infty} P(T) d T=1$, each scaled diffracted wave energy field for each period component of the spectrum can be linearly superposed to form the mean diffracted wave field for the full JONSWAP period spectrum. A spectral resolution of one second was chosen because sensitivity studies showed that using a higher period resolution only provides a very small amount of additional smoothing and has no appreciable effect on the trend of energy redistribution. 
A complete representation of the mean wave diffraction patterns under field conditions is achieved when the directional spread is convolved with the frequency distribution given above. The statistical distribution of wave directions for a random wave climate can be described, for example, with the cosine power distribution as used in the SWAN wave model so that the directional spread distribution is described by;

$$
G(\Theta)=\cos ^{\mathrm{m}}\left(\Theta-\Theta_{\text {mean }}\right)
$$

where $\Theta_{\text {mean }}$ is the mean wave direction and $m$ is the cosine power spreading parameter.

This directional spread distribution was chosen for easy comparison against the results of Miller (2007) and Black (2007). The spread function $G(\theta)$ can be scaled to a probability density function, by;

$$
D(\theta)=G(\Theta) / \int_{0}^{180} G(\Theta) d \Theta
$$

where; $1 / \int_{0}^{180} G(\Theta) \Theta$ is the normalising coefficient

The wave energy field for each integer degree of the direction spread was calculated and multiplying this by the directional spread probability function $D(\Theta)$ for that angle gives the scaled diffracted wave energy field for that angle component. As $\int_{0}^{180} D(\Theta) d \Theta=1$, each scaled diffracted wave energy field for each angle can be linearly superposed to give the mean diffracted wave field for the full directional spread. A directional resolution of one second was chosen because sensitivity studies showed that no appreciable difference is achieved using a higher resolution.

A single wave height is used for all calculations as diffraction is not a function of wave height for linear theory. For a realistic device the reflection and transmission coefficients would be a function of wave height. As such, a wave height distribution would need to be considered in the similar manner as the wave frequency distribution and directional spread distribution when the wave height dependence of the reflection and transmission coefficient for a device is determined.

\section{Model Validation}

The following shows a comparison of the disturbance coefficient predicted by the approximate analytical solution and the BEM solution in the lee of 5 overtopping type wave energy converters along the domain transects $y=5 \lambda, y=50 \lambda, y=150 \lambda$, and $x=0 \lambda$. The WEC array is located along the plane $y=0 \lambda$ and the middle of the central device is at $x=0 \lambda$. The length of each devices is $B=250[\mathrm{~m}]$ the tip to tip separation distance between neighbouring devices is $b=600[\mathrm{~m}]$, the period is discreet at $T=8[\mathrm{~s}]$, water depth is uniform at $h=50[\mathrm{~m}]$, the wavelength is $\lambda=100[\mathrm{~m}]$ (from the dispersion relationship) and this is used to normalise the dimensions of the calculation domain, the wave height is discreet and small enough for the linear theory to be applicable. The incident wave directions are $\Theta=50,70$ and $90^{\circ}$ and the permeability parameter used with the BEM solution is $\beta=0$ (full reflection zero transmission) and $\beta=k(1-i) / 4$ (partial reflection, transmission and dissipation), which translates to $c_{\rho}=1, c_{\iota}=0$, and $c_{\rho}=0.6+0.2 i, c_{\iota}=0.4-0.2 i$, respectively for use with the approximate analytical solution. The computational domain is $25[\mathrm{~km}]$ down-wave and $10[\mathrm{~km}]$ wide which equates to $250 \lambda$ long and $100 \lambda$ wide, when normalised by the wavelength.

In the moderate to far-field $(y>50 \lambda)$, which is our primary area of interest for this study, the approximate analytical solution is in excellent agreement with the mathematically exact BEM method of McIver (2005). At distance closer to the breakwater array the two solutions diverge and this is significant in some regions (primarily in the regions outside the geometrical shadow of the array). The divergence between the two methods enhances when the incident angle is more oblique or when the permeability increases. As such, we can state that the approximate analytical solution is sufficiently accurate at predicting the disturbed wave field in all but the region relatively close to the plane of array. The approximate analytical solution is suitable for scaling the farfield wave energy shadow and re-distribution but the BEM method would be required for the near-field accuracy required for spatial array optimisation studies. This result is particularly useful for this study because the approximate analytical solution is sufficiently computationally efficient to assess a large domain for a high resolution random sea state and will be useful in the future for considering the full variability of a wave climate 
case study and when we account for a wave height distribution and the dependency of wave height on the energy conversion.

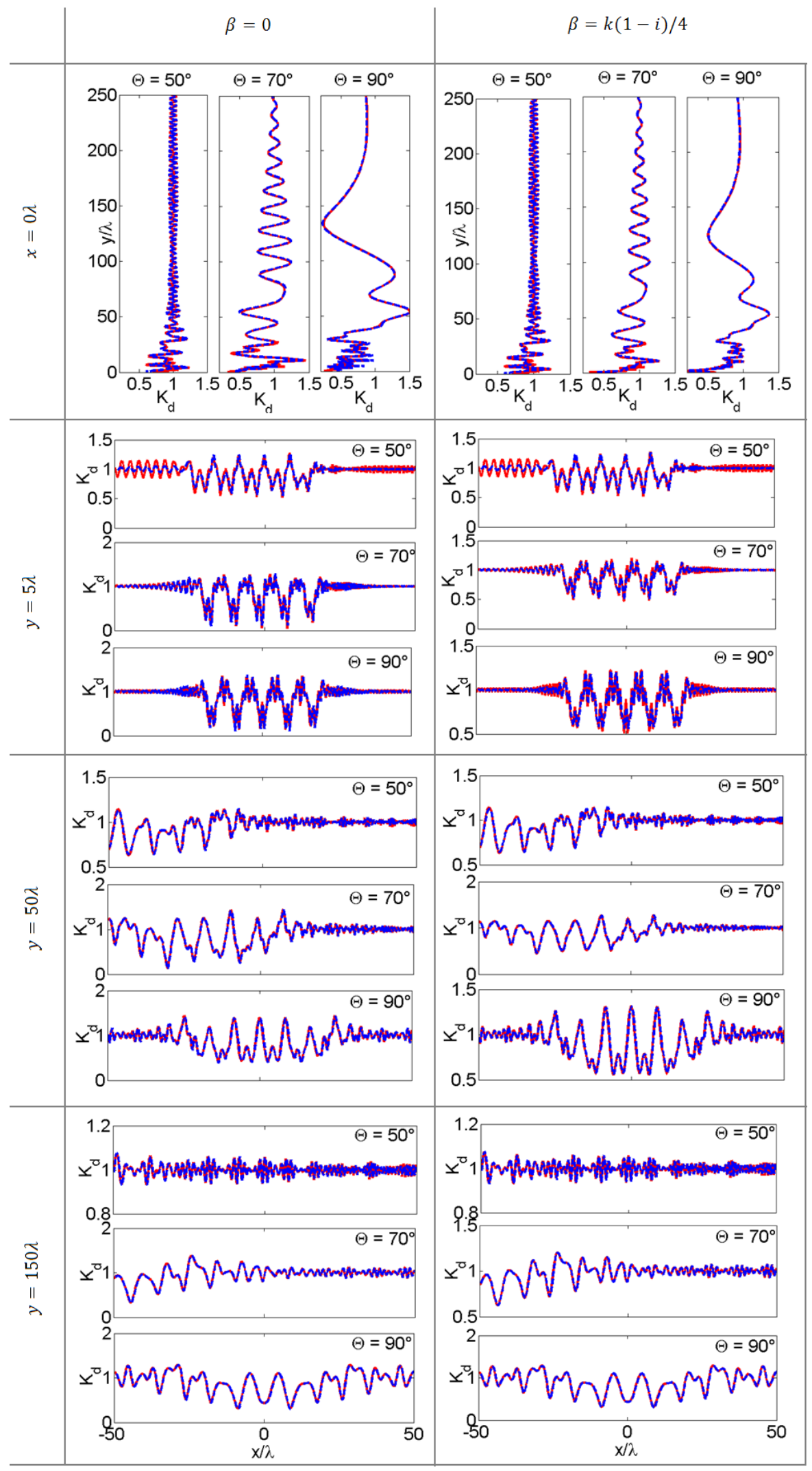


Figure 1. Comparison of disturbance coefficient $K_{d}$ for the approximate analytical solution (red line) and BEM solution (blue dashed line) along the transects; $y=5 \lambda, y=50 \lambda, y=150 \lambda(+y$ is down-wave distance from the plane of the array), and $x=0 \lambda$ ( $x=0$ is the centre of the of the device array), for incident wave directions of $\Theta=50^{\circ}, 70^{\circ}$ and $90^{\circ}$ and permeability parameters of $\beta=0$ (solid full reflection) and $\beta=k(1-i) / 4$ (partial reflection, transmission and dissipation)

\section{Results}

In this section we compare the disturbed wave field predicted by the approximate analytical solution and the no-diffraction solution. We also compare the re-distribution of energy in the "direct lee region" which we define as being the region between a half gap length before the first device in the series to half a gap length past the last device in the series. In all cases in this section the WEC array is located along the plane $y=0 \lambda$ and the middle of the central device is at $x=0 \lambda$. The length of each devices is $B=250[\mathrm{~m}]$ the tip to tip separation distance between neighbouring devices is $b=600[\mathrm{~m}]$, for the monochromatic cases the period is discreet at $T=8[s]$, and for the spectral cases $T_{p}=8[s]$ and the profile is of the JONSWAP spectrum with a peak enhancement of $\gamma=3.3$, water depth is uniform at $h=50[\mathrm{~m}]$, the wavelength is $\lambda=100[\mathrm{~m}]$ (from the dispersion relationship) for the monochromatic cases and peak wavelength is $\lambda_{p}=100[\mathrm{~m}]$ for the spectral cases and either of these are used to normalise the dimensions of the calculation domain, the wave height is discreet and small enough to be applicable to linear theory as we are not considering the dependency of wave height on energy extraction. The incident waves are either unidirectional or directional with directional spread parameters of $m=2,10,100$ and 800. The reflection coefficient is $c_{\rho}=0$ and the transmission coefficient is $c_{\iota}=0$ so that the devices are considered to be unrealistic perfect extractors/converters. The computational domain is $25[\mathrm{~km}]$ down-wave and $10[\mathrm{~km}]$ wide which equates to $250 \lambda$ and $100 \lambda$ when normalised by the peak wavelength. This is a relevant domain size as the down wave distance from the wave hub project to the coast is approximately $25[\mathrm{~km}]$. Fig. 2 shows the disturbed wave field predicted by the approximate analytical solution. When the incident waves are considered to be monochromatic and unidirectional a strong interference pattern is present. When spectral waves are considered, the spatial locations of the maxima and minima interference pockets changes for each discreet component of the frequency distribution and the result is a smoothing of the interference pattern. When directional spreading is considered strong smoothing of the interference pattern occurs and the wave energy shadow is seen (on the most part) to recover with distance from the array with a greater directional spread resulting in a faster rate of recovery. Fig. 3 shows the disturbed wave field as predicted by the no-diffraction solution. As diffraction is not considered, the wave energy shadow for both monochromatic unidirectional, and spectral unidirectional incident waves, is simply a projection of the geometrical shadow of the devices. When directional spreading is accounted for we see a similar smoothing and regeneration of the wave energy shadow with increasing distance from the array which is more rapid with a greater directional spread (lower directional spread cosine power $m$ ).

As the mean incident wave direction is perpendicular to the array the greatest reduction in wave height will generally be along the transect that bisects the central device of the series "central transect" because this is the point at which the recovery of the wave energy shadow from directional spreading is at its slowest (although local interference will modify this in some regions). As seen in Fig. 4 there are some appreciable differences between predictions of the approximate analytical solution and the no-diffraction solution for the disturbance coefficient along the central transect. When diffraction is considered the narrower directional spreads of $m=100$ and 800 show a divergence in wave height so that the no-diffraction solution shows greater wave heights with increasing distance from the array (when $m=800$ there is a temporary interference maxima along the central transect near the array which provides a greater wave height at first compared to the no-diffraction solution). This is counter-intuitive as diffraction is often considered to be a mechanism that reduces the lateral wave height gradients so one might expect that diffraction would act to redistribute wave energy from the high region (the region outside the geometrical shadow of the array) to the low energy region (the region inside the geometrical shadow of the array), thus helping the wave energy shadow to recover more rapidly with distance from the array. 

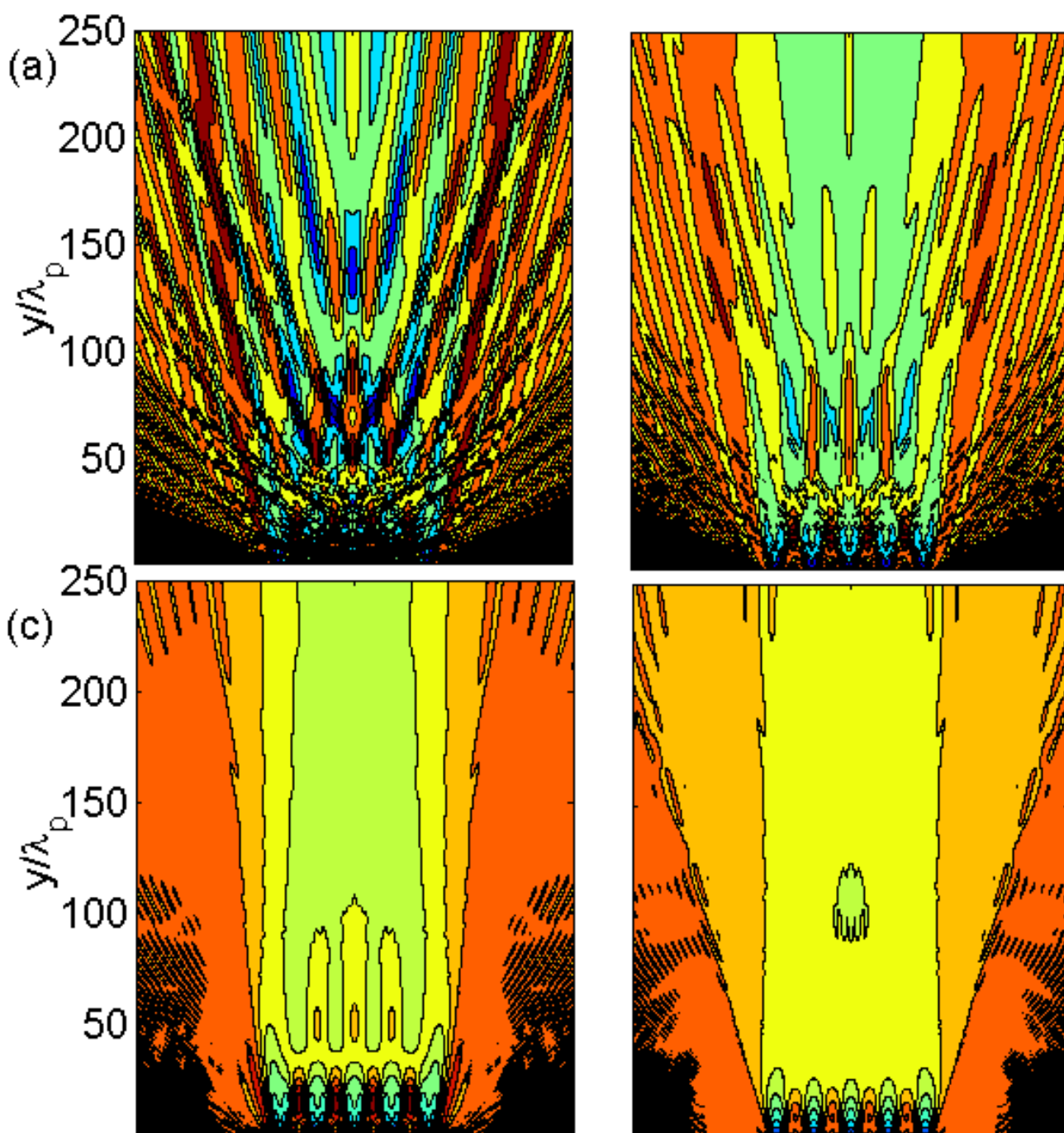

(b) $K_{d}$

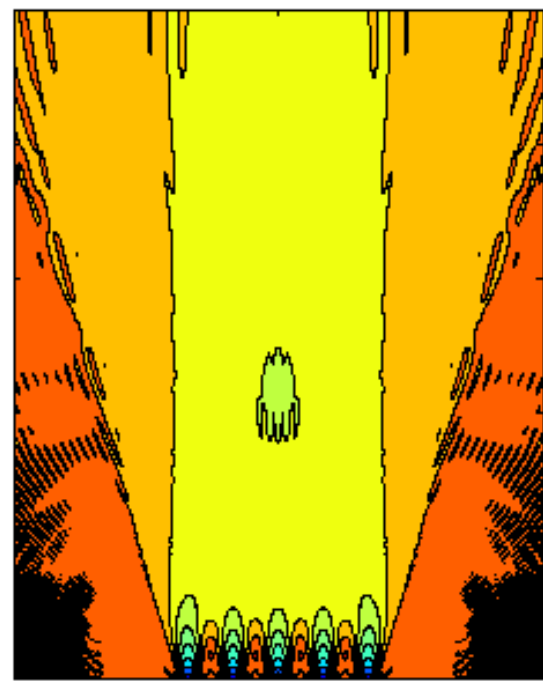

(d)

(e)
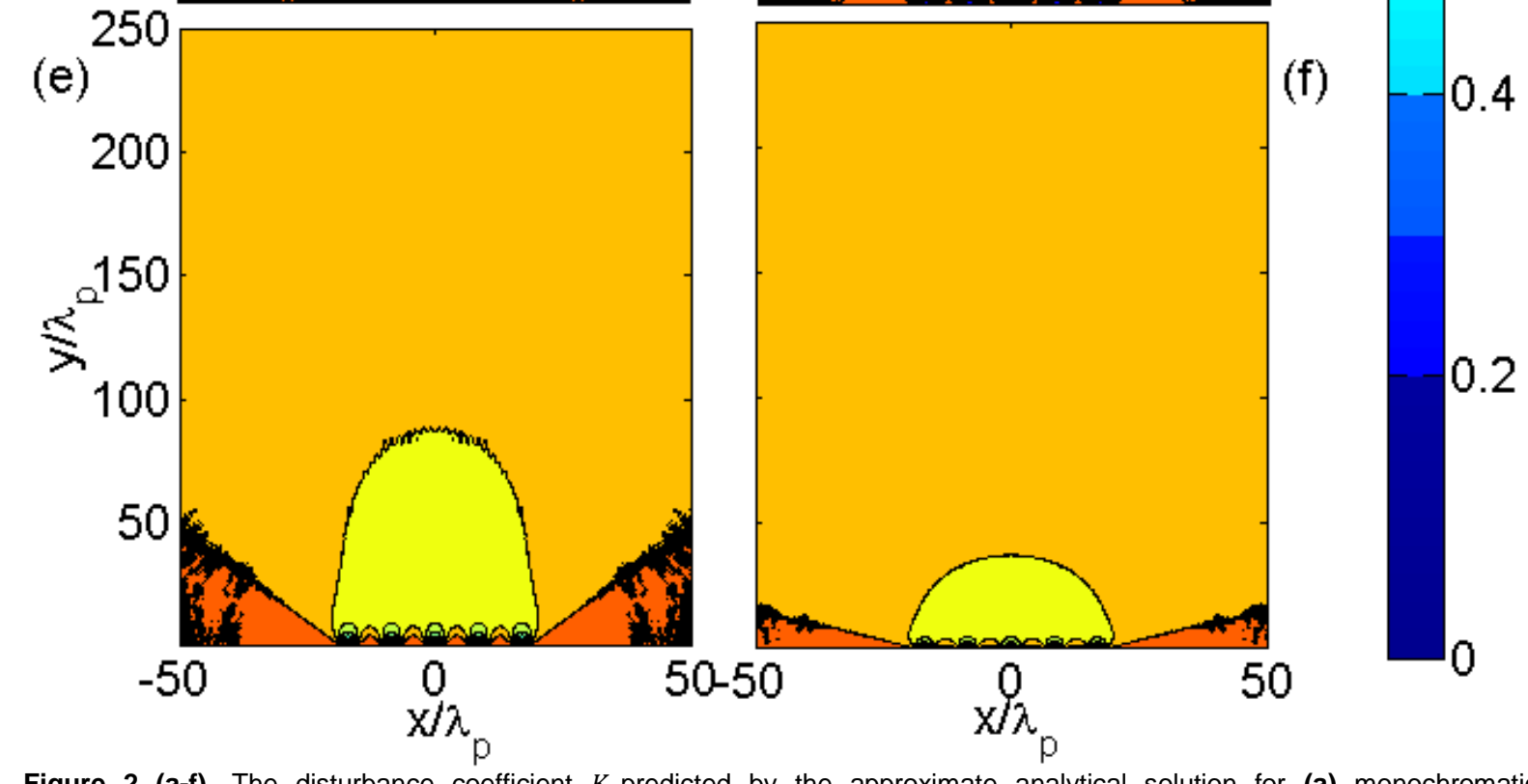

(f)

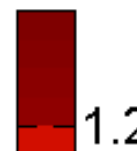

1.2

Figure 2 (a-f). The disturbance coefficient $K_{d}$ predicted by the approximate analytical solution for (a) monochromatic unidirectional incident waves, (b-f) are spectral waves with $T_{p}=8[s]$ and peak enhancement $\gamma=3.3$, with (b) unidirectional, (c) directional with $m=800$ (d) directional with $m=100$,(e) directional with $m=10$, (f) directional with $m=2$. In all cases the mean wave direction is $\Theta=90^{\circ}$ from the plane of the array and $c_{\rho}=0$ and $c_{\iota}=0$. 


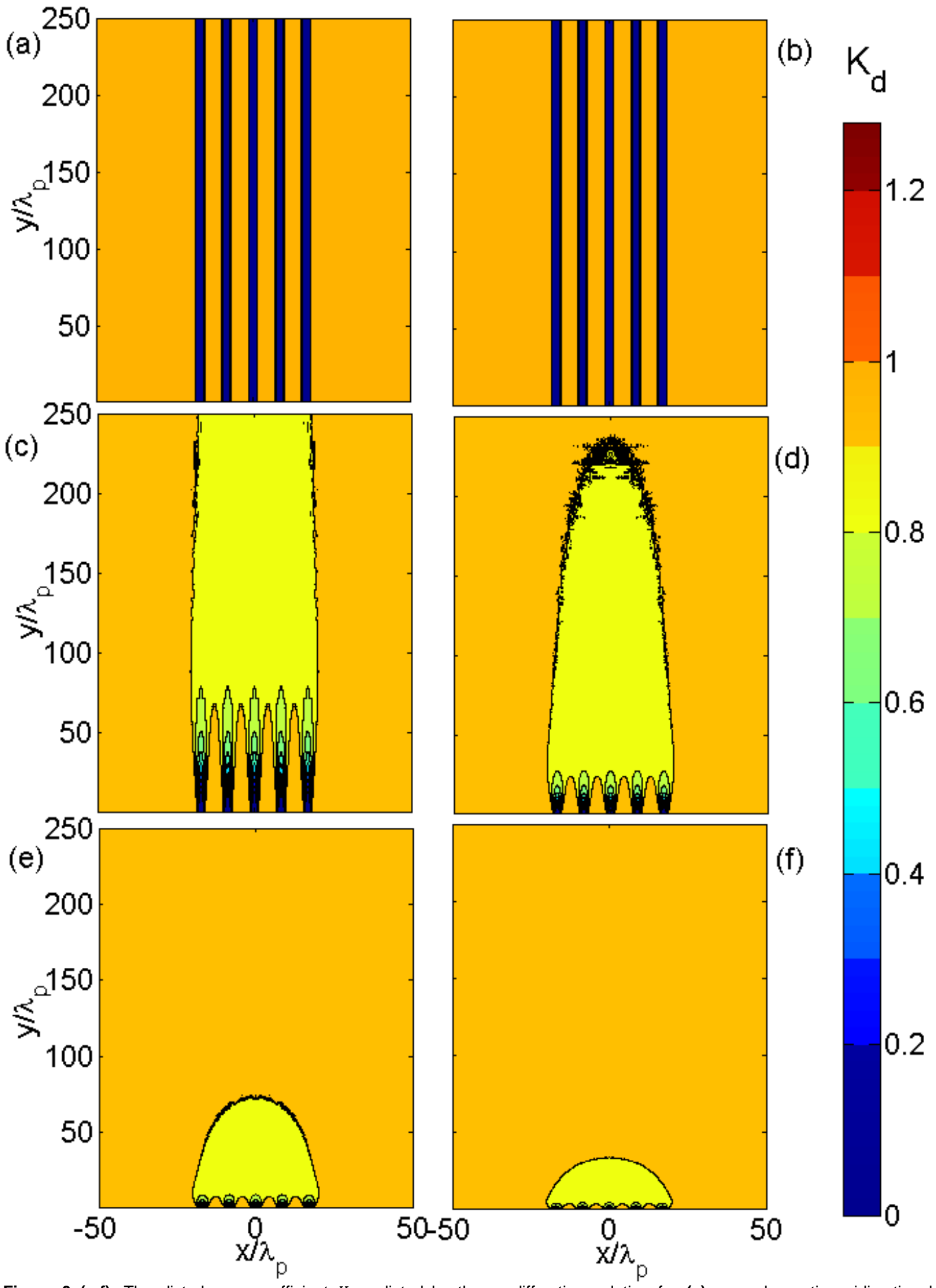

Figure 3 (a-f). The disturbance coefficient $K_{d}$ predicted by the no-diffraction solution for (a) monochromatic unidirectional incident waves, (b-f) are spectral waves with $T_{p}=8[s]$ and peak enhancement $\gamma=3.3$, with (b) unidirectional, (c) directional with $m=800$ (d) directional with $m=100$,(e) directional with $m=10$, (f) directional with $m=2$. In all cases the mean wave direction is $\Theta=90^{\circ}$ from the plane of the array and $c_{\rho}=0$ and $c_{\iota}=0$. 
(a)

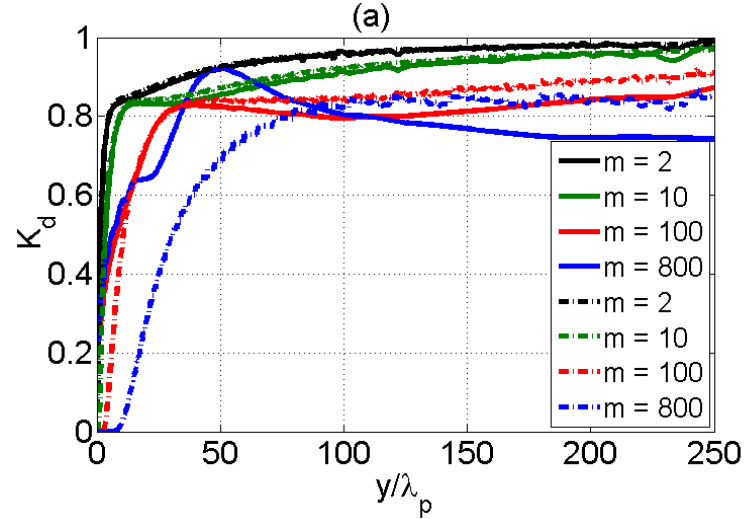

(b)

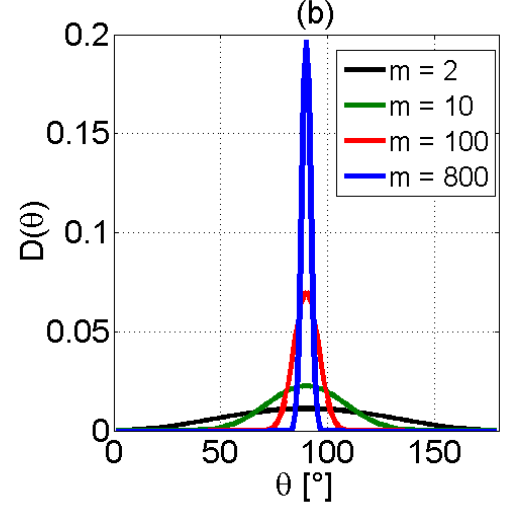

Figure 4 (a-c). (a) The disturbance coefficient $K_{d}$ along the transect that bisects the central WEC device of the series predicted by the approximate analytical solution (solid line) and the no-diffraction solution (dot dashed line). (b) shows the wave directional spread probability density for discreet angular components of one degree.

To assess the net effect of diffraction on the re-distribution of wave energy we averaged the wave energy in each band of the domain $\overline{K_{d, y}{ }^{2}}$ in the "direct lee region" $(y=0[m], 25[m] . .25000[m])$ this is then normalised by the incident undisturbed wave energy $E_{0}$ so that the average energy disturbance coefficient in that band is given by;

$$
E_{d, y}=\overline{K_{d, y}{ }^{2}} / E_{0}
$$

Interestingly, the wave energy in the "direct lee region" for narrower directional spreads $(m=$ $100,800, \infty)$ is less when diffraction effects are considered as seen in Fig.5. Also for the case of a very narrow directional spread $(m=800)$ the total energy in each band of the domain gets progressively less with increasing distance from the array. It would appear that the diffraction effect spreads energy from the high energy regions outside the geometrical shadow into the low energy region in the geometrical shadow of the array, initially, but that this lateral re-distribution of energy overshoots the "direct lee region", propagating energy back into the open regions on the opposing side of the array. As the diffraction wave components of the approximate analytical solution are weighted radiation waves, the effect of the devices is to causes a portion of the incident plane parallel wave to become radial waves thus defocusing wave energy from the geometrical shadow of the array for that incident wave direction. When wave directional spreading is broad the diffraction effect defocuses wave energy from the "direct lee region" for the incident angles that are close to the mean direction but this is countered by the more oblique indecent wave angles of the distribution where diffraction defocuses wave energy from geometrical shadow area for the broader angular components back into the geometrical shadow for the mean direction. The end result is that the re-distribution of wave energy from diffraction has a negative effect on the recovery of the wave energy shadow but only when the wave directional spread is narrow. Specifically fwebsiteor the case when the directional spread parameter is $m=800$ the difference in average wave energy in the "direct lee region" is $12 \%$ of the incident undisturbed wave energy when $m=100$ this reduces to $5 \%$ and there is no appreciable difference when $m=10$ and 2 .

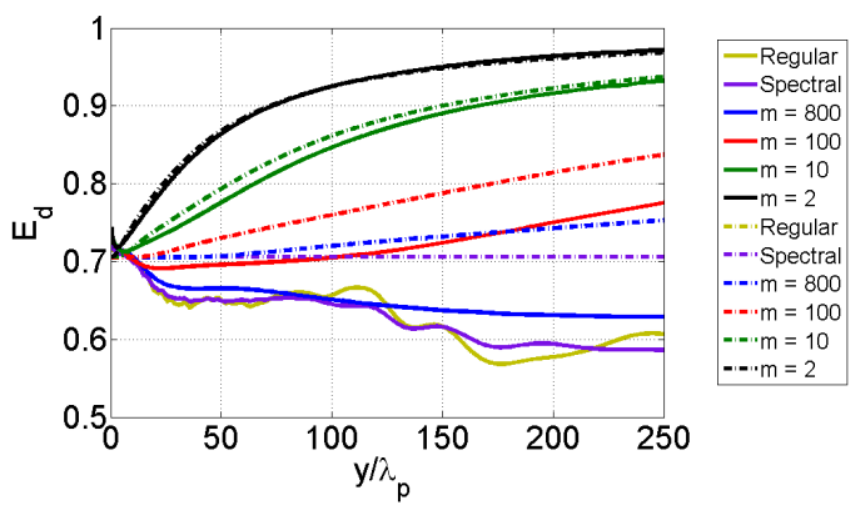

Figure 5 (a-b). The change in total wave energy for each band of the "direct lee region" with increasing distance from the array for unidirectional monochromatic waves with $T=8[\mathrm{~s}]$, unidirectional spectral waves with $T_{p}=8[\mathrm{~s}]$, and directional spectral 
waves with $T_{p}=8[s]$ and spread parameters $m=2,10,100$ and 800 . Solid lines show the approximate analytical solution and dot dash lines show the no-diffraction solution.

\section{Discussion}

The objective of this study is to examine how diffraction re-distributes wave energy with increasing downwave distance from the array. To investigate this effect without confusing this with other processes we have only considered an array of simplified perfect overtopping type WEC devices. The devices are considered to be un-realistic perfect energy extractors across all wave heights, wave periods and incident directions. In reality the extraction of the wave energy incident on the device will be much less than considered here with some energy being reflected and transmitted and this will be strongly dependent on the incident wave parameters. Each incident wave direction, frequency and wave height permutation will have a specific power take off associated with it. With this methodology in place and when the device's specific energy extraction is known. it would be straight forward to consider more realistic devices so that a more realistic wave energy shadow could be predicted. Other effects such as refraction, friction, wind and shoaling should also be considered to assess the change in wave climate at the coast and the impact of these processes will depend on the specific case study. However the method and solution presented here provides a useful tool for scaling the potential wave energy shadow in the far field that would be expected from an array of overtopping type WEC devices.

\section{Conclusions}

The approximate analytical solution presented here provides a method for assessing the disturbed wave field about a series of reflecting, transmitting and dissipating breakwater segments, which we consider to be approximations of overtopping type wave energy converters. The solution is in excellent agreement with the boundary element method of McIver (2005) except for the region close to the array. The advantage of the approximate analytical solution over the BEM method is that it is computationally efficient so large domains can be considered for high resolution incident irregular sea states on a desktop PC. The method is particularly useful when assessing the wave energy shadow for a WEC array far offshore, when a full wave climate is to be assessed, or the full evolution of the wave energy shadow is under investigation (as in this study). The approximate analytical solution also permits strong energy dissipation in the breakwater which we use to approximate wave energy conversion by the overtopping type WEC device.

The diffraction effect was found to defocus wave energy from the geometrical shadow of the array for the specific incident wave direction because the incident plane wave becomes, in part, a radial wave propagating energy away from the incident wave direction. For very narrow directional spreads the result is opposite to the recovery of the wave energy shadow from directional spreading so that the wave energy shadow persists for longer distances from the array and even gets deeper for very narrow directional spreads. For broader directional spreads there is no-difference in the wave energy shadow with and without including diffraction, for the specific arrangement of devices and incident wave parameters that we consider. This is because the defocusing of energy for the strongly oblique wave components counters the defocusing of energy for the wave components closer to the mean direction.

\section{ACKNOWLEDGMENTS}

The first author would like to acknowledge the PhD studentship from Plymouth University that has funded this research. The second author would like to acknowledge the support of the start-up fund by University of Maine.

\section{REFERENCES}

Beels, C., Troch, P., De Visch, K., Kofoed, J.P. and De Backer, G., 2010. Application of the time-dependent mild-slope equations for the simulation of wake effects in the lee of a farm of wave dragon wave energy converters. Renewable Energy, 35(8), 1644-1661.

Black, K., 2007. Review of wave hub technical studies: Impact on inshore surfing beaches. ASR ltd.

Daemrich, K.-F. and Kohlhase, S., 1978. Influence of breakwater reflection on diffraction. Proceedings of the 16th International Conference on Coastal Engineering. ASCE, 651-663

Hotta, S., 1978. Wave height distribution around permeable breakwaters. Proceedings of the $16^{\text {th }}$ International Conference on Coastal Engineering. ASCE, 695-714

Kim, S.D. and Lee, H.J., 2010. The comparison of analytical and numerical solutions for wave diffraction due to insular breakwater. International Journal of Physical Sciences, 5(3), 226-237.

McCormick, M.E. and Kraemer, D.R.B., 2002. Polynomial approximations for fresnel integrals in diffraction analysis. Coastal Engineering, 44(3), 261-266. 
Millar, D.L. Smith, H.C.M. and Reeve, D.E., 2007. Modelling analysis of the sensitivity of shoreline change to a wave farm. Ocean Engineering, 34(5-6), 884-901.

Mitsui, H. and Murakami, H., 1967. Wellenhöhenverteilung an diskontinuierlichen teilen von kustenbauwerken. Kaigan Kogagu Koenkai Koenshu 14

Monk, K. Zou, Q. Conley, D. 2012a. An approximate solution for the wave energy shadow in the lee of an array of overtopping type wave energy converters. Costal engineering. under review

Monk, K.U., Zou, Q.-P., and D. C. Conley 2012b. Numerical and Analytical Simulations of Wave Interference about a Single Row Array of Wave Energy Converters, Estuary and Coast, submitted.

Nørgaard, J. H. and Andersen, T. L., 2012. Investigation of Wave Transmission from a Floating Wave Dragon

Wave Energy Converter, Proceedings of the $22^{\text {nd }}$ International Offshore and Polar Engineering Conference, 509-516.

Ou, Shan-Hwei., Tzang. Shiaw-Yih., Hsu. Tai-Wen., 1988. Wave field behind the permeable detached breakwater, Proceedings of the $21^{\text {st }}$ International Conference on Coastal Engineering.

Penney, W.G. and Price, A.T., 1952. The diffraction theory of sea waves and the shelter afforded by breakwaters. Phil. Trans. R. Soc, (Ser. A 244), 236-253.

Silvester, R. and Lim, T.-K., 1968. Application of wave diffraction data. Proceedings of the 11th International Conference of Coastal Engineering, ASCE, 248-270

Sommerfeld, A., 1886. Mathematische theorie der diffraction. Math Annalen, 47, 317-374.

Venugopal, V. and Smith, G.H., 2007. Wave climate investigation for an array of wave power devices. 7th European wave and tidal energy conference.

$\mathrm{Yu}, \mathrm{X}$. and Togashi, H. 1996. Combined diffraction and transmission of water waves a around porous breakwater gap. Proceedings of the 25th International Conference of Coastal Engineering. ASCE, 2063-2076 